\title{
Cuota de género y sistemas electorales: Argentina y Brasil en perspectiva comparada
}

Gender quota and electoral systems: Argentina and Brazil in comparative perspective

\section{Joaquina Altamirano}

Universidad Católica de La Plata, Argentina

joaquina.a@hotmail.com

Julieta Mulet

Universidad Católica de La Plata, Argentina

mulet.julieta@gmail.com

\section{RESUMEN:}

El presente trabajo tiene por objetivo evaluar el impacto efectivo de las leyes de cuota de género en Argentina y Brasil respecto de la finalidad para la cual fueron sancionadas, a saber, lograr un incremento de la presencia femenina en los parlamentos, considerando además las condiciones en que dicha participación tiene lugar, estrechamente ligada al sistema electoral de ambos países. Cuestionar el sistema electoral, como construcción que impacta en la dinámica política, es un primer paso para avanzar en el análisis de la participación y el acceso de las mujeres a espacios de poder al interior de nuestras democracias. Con tal motivo se evaluarán distintas subdimensiones del propio sistema, tales como el sufragio, la conversión de votos en escaños, las candidaturas, el tamaño y la composición de la Asamblea y la estructura de las cuotas de género establecidas legalmente. Para ello serán considerados tres momentos electorales legislativos de cada uno de los dos países: las elecciones previas a la sanción de la ley de cuota, las elecciones inmediatamente posteriores a la norma y las últimas elecciones.

Palabras Clave: Cuota de género, Sistemas Electorales, Análisis Comparativo, Argentina, Brasil.

\section{Abstract:}

The aim of this paper is to evaluate the effective impact of gender quota laws in Argentina and Brazil vis-á-vis the purpose for which they were enacted, namely, to achieve an increase in female presence in Parliaments, taking into account the conditions in which such participation takes place, closely linked to the electoral systems of both countries. Conceiving the electoral system as a construction impacting on political dynamics is a first step to later consider the analysis of women's participation and access to power spaces within our democracies. For this reason, different subdimensions of the system will evaluate, such as the suffrage, the conversion of votes into seats, the candidatures, the size and composition of the Assembly and the structure of legally established gender quotas. Three legislative electoral moments of each of the two countries will be considered: the elections prior to the enacting of the quota law, the elections immediately following the norm and the last elections.

KEYWORDS: Gender Quota, Electoral System, Comparative Analysis, Argentina, Brazil.

\section{INTRODUCCIÓN}

El creciente espacio ganado por el movimiento feminista latinoamericano en las agendas sociales, culturales, mediáticas, e institucionales, no sólo ha logrado visibilizar diversos temas de las mujeres, ${ }^{1}$ sino además ha afirmado el debate en torno a sus roles en la sociedad y su relación con los espacios de poder. Preguntarse por la participación de las mujeres en dichos espacios supone pensar la manera en que se participa y el grado de representación del colectivo de género que ello trae aparejado, así como también poner en cuestión los mecanismos que existen para ello. Los resultados arrojados por una realidad que nos interpela, motivan el interés por las cuotas de género que supone observar más allá de la ley y al interior del sistema electoral para comprender su impacto en la distribución del poder. 
La década del '90 fue escenario de debates sobre la aplicación de leyes de cuota en América Latina, siendo Argentina pionera en establecer regulaciones en este campo y constituyendo así un modelo para el resto de los países que conforman la región. Las cuotas legales aparecieron por entonces como respuesta al debate sobre las formas en que podía asegurarse un mínimo de participación femenina en la arena política, más concretamente en las contiendas electorales (Archenti y Tula, 2007; Marx, Borner y Caminotti, 2007). Entender el contexto en que estas reivindicaciones tienen lugar es crucial para comprender el proceso de forma integral: la redemocratización devolvió a ciudadanos y ciudadanas el derecho de participar de la vida política tras años de dominación autoritaria, al mismo tiempo que la herencia recibida de los años precedentes y la carencia de una firme tradición democrática alimentaba la reproducción de formas hegemónicas de dominación y opresión, contrarias a la esencia de las ideas de democracia, participación e igualdad.

La elección de Argentina y Brasil como unidades de análisis para esta investigación, producto de un trabajo exploratorio inicial, partió no sólo de la centralidad que tienen en el Cono Sur, sino además de entender que en ambos países han tenido lugar sucesos históricos relacionados con el vínculo mujeres-poder.

En el último decenio, la Casa Rosada y el Palacio de Planalto fueron simultáneamente sede de fuertes liderazgos femeninos. Las presidencias de Cristina Fernández de Kirchner y Dilma Rousseff son reflejo de una tendencia creciente de acceso por parte de las mujeres al ejercicio de cargos públicos en general, y ejecutivos en particular, no siendo un detalle menor el hecho de que en ambos casos se trata de acentuados presidencialismos, en donde la figura presidencial, históricamente ocupada por hombres, es central.

En la actualidad, el acceso de las mujeres a espacios de poder tradicionalmente androcéntricos "coloca a las líderes, aunque no se lo propongan, en el sitio simbólico y político de la representación colectiva de género" (Castañeda, 2013, p. 26) lo cual las sitúa frente al desafío de redefinir las pautas de acceso al poder y de configurar una agenda que sea más representativa de las demandas de las mujeres en la vida política, económica, social y cultural (Marx, Borner y Caminotti, 2007).

Si se entiende la participación como "tomar parte en persona, y una parte autoactiva" (Sartori, 1988, p. 153), hablar de participación efectiva está orientado a las posibilidades reales que tienen las mujeres de acceder a espacios de poder. La representación, por su parte, se vincula a "hacer presente en algún sentido algo que, sin embargo, no está presente literalmente o de hecho" (Pitkin, 1985, citado en Marx et al., 2007, p. 201), una nota característica de cualquier democracia representativa.

A su vez, Hanna Pitkin (1985) propone una división del concepto de representación que resulta de interés para el abordaje de nuestro objeto de estudio. Por un lado, introduce la idea de representación descriptiva, que implica la correspondencia o semejanza precisas respecto de aquello que se representa, dependiendo así la misma de las características del representante, lo que es o parece ser, antes de lo que hace; y por otro, la representación sustantiva que hace a actuar en interés de quienes se representa. Al seguir esa línea teórica, la mera presencia de las mujeres en espacios de representación política se vincula al primero de estos tipos de representación mientras que la representación sustantiva de género guarda relación con la promoción de los intereses de las mujeres, concentrando la actuación de los y las representantes en su responsabilidad ante la ciudadanía, mientras que sus características personales no son relevantes (Caminotti, 2013).

La realidad pone en evidencia la tensión que existe entre la participación y la representación y, a la vez, entre representación descriptiva y sustantiva. La ocupación de espacios de poder por las mujeres no tiene como correlato automático la representación de los intereses del heterogéneo colectivo femenino porque la participación no supone un mandato imperativo de representación del propio género y, mucho menos, de rendición de cuentas al mismo.

La legislación como resultado de un proceso de construcción social dinámico tiene fuerza correctiva sobre las desigualdades que atraviesan las sociedades (Sartori, 2014). Las leyes de cuota de género se insertan en esa lógica, pero la dimensión legal de las cuotas no es el único aspecto legislativo de las mismas; hablar de las leyes de cuota de género es también hablar de cómo se legislará a partir de su puesta en vigencia. 
La participación efectiva de las mujeres en la arena política institucional, en general, y en el área legislativa, en particular, es en parte condicionada por dimensiones del sistema electoral. Con el objetivo de evaluar el impacto de las cuotas de género en Argentina y Brasil, el presente trabajo abordará los sistemas electorales de ambos países a través de distintas dimensiones, tales como el sufragio, la candidatura, la conversión de votos en escaños, la Asamblea y la estructura de cuotas de género, considerando además tres momentos electorales legislativos de cada uno de los dos países, a saber, las elecciones previas a la sanción de la ley de cuota, las elecciones inmediatamente posteriores a la norma y las últimas elecciones. ${ }^{2}$

\section{Voz y voto: Mujeres, Cuotas y Sistemas electorales de Argentina y Brasil}

El binomio mujer-poder continúa despertando cuestionamientos y dudas aun en el siglo XXI. Dudas que, como recurso epistemológico político, dan pie a cuestionar lo establecido y, con ello, a pensar como colectivo formas alternativas de actuar y pensar la relación de las mujeres y el poder, la sociedad, el Estado (Castañeda, 2013). En este sentido, cuestionar el sistema electoral, y con ello las leyes de cuota, es un primer paso para avanzar en el análisis de la participación política y el acceso de las mujeres a espacios de poder al interior de nuestras democracias.

Hablar de sistema electoral implica hacer referencia al modo en que los electores manifiestan a través del voto el partido o candidato/a de su preferencia y la forma en que esos votos se convierten en escaños (Nohlen, 1994). De este modo, el sistema electoral, como articulación del régimen electoral (es decir, de todos los aspectos normativos que regulan el proceso) con las elecciones en sí mismas, posee diversas subdimensiones que impactan no sólo en la configuración del propio proceso electoral y el lugar que ocupan en éste los distintos actores, sino además, en el resultado electoral y en el propio sistema político.

\section{1. El sufragio}

Las elecciones libres, periódicas, competitivas y transparentes entendidas como técnica de designación de representantes a través del sufragio popular universal, igual, secreto, directo y libre, son la forma más importante de participación política institucionalizada en la que se manifiestan en menor grado las distorsiones socioculturales y, a la vez, condición sine qua non de los sistemas democráticos en los Estados modernos, aunque no por ello suficientes ni exclusivas de este tipo de regímenes (Dahl, 1992; Nohlen, 1994; Sartori, 1988).

Actualmente, tanto Argentina como Brasil consagran en sus legislaciones el derecho universal al sufragio; sin embargo, no siempre fue así.

En el año 1912 fue sancionada en Argentina la Ley $N^{\circ}$ 8.871, más conocida como Ley Sáenz Peña, que estableció el sufragio universal masculino, secreto y obligatorio desde los 18 años. Brasil debió esperar dos décadas para la ampliación del derecho al sufragio; sin embargo, el Código Electoral de 1932 extendía el mismo tanto a hombres como a mujeres mayores de 18 y 21 años de edad, respectivamente. El reconocimiento de los derechos políticos de las mujeres en Argentina tendría lugar finalmente en la década del cuarenta con la sanción de la Ley $\mathrm{N}^{\circ} 13.010$.

Los períodos autoritarios en la región durante la segunda mitad del siglo XX impidieron la expresión material de lo consagrado en las normas. Así, la consolidación electoral tardía en ambos países responde a la juventud de su estabilidad democrática. Desde la redemocratización en los años ' 80 hasta nuestros días, las elecciones se han celebrado de manera ininterrumpida y se han introducido modificaciones en la legislación electoral. $^{3}$

Desde la vuelta a la democracia Argentina consagró el sufragio universal, igual, secreto y obligatorio a través del artículo No 1 del Código Electoral Nacional (1983) y luego con el artículo o 37 de la Constitución de la 
Nación Argentina (1994) - en adelante CEN y CNA, respectivamente-, reconocido para los ciudadanos y las ciudadanas de entre 18 y 70 años, y el sufragio opcional para mayores de 70 , siendo esta también una posibilidad para las y los jóvenes desde los 16 años con la sanción de la Ley $\mathrm{N}^{\circ} 26.774$ en 2012.

El Código Eleitoral de 1965 (en adelante, CEB), en su artículo 82, y la Constitución de Brasil de 1988 (en adelante, y CNB), sancionada tres años después de iniciada la transición democrática, en su artículo 14, reconocen el sufragio directo, secreto, universal e igual para los ciudadanos y las ciudadanas brasileñxs.

Evaluar el sufragio en tanto subdimensión del sistema electoral, no se agota en la referencia a quiénes tienen el derecho de ejercer el voto, sino que supone ampliar el horizonte de análisis a variables cuantitativas que den cuenta de aspectos de demografía electoral tales como la cantidad de habitantes habilitados para votar, la composición por género del voto y los niveles de participación electoral efectiva. De acuerdo con los datos oficiales obtenidos y plasmados en el Cuadro 1, la demografía electoral de Argentina y Brasil evidencia un proceso de expansión en el marco temporal de este trabajo.

\section{CUADRO 1}

\begin{tabular}{|l|l|l|l|l|l|l|l|}
\hline \multicolumn{2}{|l|}{$\begin{array}{l}\text { ARGENTINA } \\
\text { Año electoral }\end{array}$} & $\mathbf{1 9 9 1}$ & $\mathbf{1 9 9 3}$ & $\mathbf{2 0 1 7 4}$ & $\mathbf{1 9 9 4}$ & $\mathbf{1 9 9 8}$ & $\mathbf{2 0 1 4}$ \\
\hline $\begin{array}{l}\text { Cantidad de } \\
\text { habitantes } \\
\text { habilitados para } \\
\text { votar }\end{array}$ & 20.764 .012 & 21.463 .761 & 31.082 .401 & 94.782 .803 & 106.053 .106 & 142.822 .046 \\
\hline $\begin{array}{l}\text { Cantidad de } \\
\text { habitantes } \\
\text { habilitados } \\
\text { para votar } \\
\text { por género }\end{array}$ & M F & 10.016 .484 & 10.016 .484 & 13.965 .138 & 47.681 .889 & 53.033 .650 & 68.247 .598 \\
\cline { 2 - 8 } & 10.861 .687 & 10.861 .687 & 15.207 .519 & 46.804 .055 & 52.794 .597 & 74.459 .424 \\
\hline $\begin{array}{l}\text { Participación } \\
\text { efectiva }\end{array}$ & 16.595 .952 & 17.242 .141 & 25.413 .205 & 77.944 .089 & 83.673 .537 & 115.122 .511 \\
\hline
\end{tabular}

Fuente: Cuadro de elaboración propia con base en los datos obtenidos de: Ministerio del Interior Nacional, recuperado de http://www.mininterior.gov.ar/asuntos_politicos_y_alectorales, y de Tribunal Superior Eleitoral o Tribunal da Democracia, recuperado de http://www.tse.jus.br/institucional/o-tse/o-tse.

\section{2. Las candidaturas}

Como el sistema electoral se compone de electores y elegidos, así como hablamos del sufragio, relativo a los primeros, es necesario mencionar su contraparte en el caso de lxs segundxs: las candidaturas. Al hablar de ellas, corresponde evaluar al menos dos cuestiones.

La primera tiene que ver con la existencia o no de elecciones primarias y los términos en que las mismas tienen lugar. Las elecciones primarias se corresponden con un proceso de selección de candidatxs de cara a las elecciones generales, resultando así un factor de democratización de la vida interna de los partidos políticos.

Desde el año 2009, y en función de las disposiciones de la Ley $N^{\circ} 26.571$, el sistema electoral argentino reconoce las primarias abiertas, simultáneas y obligatorias (PASO) como instancia electoral. Convocadas por el Poder Ejecutivo Nacional (art. 20), las primarias alcanzan a todas las agrupaciones políticas, aún en los casos 
en que se presentare una sola lista (art. 19). En el caso de Brasil las elecciones primarias no están reguladas por la legislación electoral del país y tampoco constituyen un método utilizado al interior de los partidos.

La segunda cuestión a tener en cuenta es la estructuración de la boleta de votación que hace a la forma en que se presenta la candidatura (Nohlen, 1994). En el caso de la elección de los miembros del poder legislativo, que es lo que interesa particularmente al presente trabajo, se emplean listas o planchas, que pueden adoptar diferentes formatos marcando distintas posibilidades de expresión de las preferencias del electorado.

Conforme el Código Electoral Nacional (1983), Argentina emplealistas cerradas y bloqueadas en sus elecciones parlamentarias con listas de dos candidatos titulares y dos suplentes en el caso del Senado Nacional (art. 156) y una cantidad determinada en función del criterio de proporcionalidad demográfica por cada una de las provincias más los correspondientes suplentes en la Cámara Baja (art. 158). Este tipo de listas suponen un alto grado de dependencia de los candidatos y las candidatas respecto de sus partidos, a la vez que no admiten modificación de ningún tipo por el electorado.

El voto en bloque es también la modalidad empleada en Brasil en ocasión de las elecciones del Senado Federal; sin embargo la Cámara de Diputados adopta un tipo de listas cerradas y no bloqueadas que permite al electorado decidir qué candidatos/as y en qué orden, dentro de los propuestos por los partidos, deben representarlos, pudiendo votar por un candidato o candidata o bien por una lista partidaria/de coalición (Nohlen, 1994), dando así mayores márgenes de maniobra a los candidatos y candidatas respecto de su estructura partidaria.

\section{3. La conversión de votos en escaños}

La conversión de votos en escaños hace a la manera en que se reparten los escaños en disputa entre lxs candidatxs que participaron de la contienda electoral, siendo así un mecanismo clave en la configuración de los resultados y, por lo tanto, con efectos políticos decisivos.

Pensar en esta cuestión remite a dos elementos fundamentales. El primero de ellos es el umbral legal, que es un mecanismo de regulación que determina un mínimo porcentual de votos que los partidos deben obtener en vistas a acceder a los cargos públicos.

He aquí una diferencia sustancial entre los países que se analizan. Mientras Brasil no presenta barreras electorales legales, Argentina tiene un umbral del 1,5\% de los votos válidamente emitidos en ocasión de las PASO (Ley N²6.571, 2009, art. 45) y del 3\% en las elecciones generales (CEN, 1983, art. 60).

El segundo factor, es la fórmula electoral, que referencia a la regla aplicada para la conversión de votos en escaños. Argentina se caracteriza por la aplicación de fórmulas proporcionales en ambas cámaras (CEN, 1983, arts. 57 y 161; CNA, 1994, art. 54), mientras en Brasil las cámaras aplican distintas fórmulas entre sí, empleando el Senado la fórmula de mayoría simple y la Cámara de Diputados Federal una fórmula proporcional (CEB, 1965, arts. 83 y 84 ; CNB, 1988, arts. 45 y 46).

\section{4. La Asamblea: su composición}

El resultado final de cualquier elección legislativa es la conformación de la Asamblea; así, esta es parte del análisis del sistema electoral porque es su resultado y ofrece variables explicativas en términos de la cantidad de escaños en disputa y cuántos de ellos son ocupados efectivamente por mujeres, permitiendo determinar el grado de proporcionalidad que encuentra la participación femenina en los parlamentos de los países en estudio.

Nélida Archenti y María Inés Tula (2007) plantean que es importante tener en cuenta el tamaño de la Asamblea en pos de un mejor entendimiento del funcionamiento de las cuotas de género. Argentina y Brasil 
establecen el tamaño de sus asambleas en sus Constituciones Nacionales y en ambos casos sus respectivos Congresos Nacionales son bicamerales (CNA, 1994, art. 44; CNB, 1988 art. 44).

En el caso de Argentina, la cantidad de diputados por el pueblo de las provincias puede aumentar (no disminuir) a partir de los censos que se realizan cada 10 años (CNA, 1994, art.45). Así se pasó de 254 miembros en 1991 a 257 en 1995, y manteniéndose tal número en la actualidad. La reforma constitucional de 1994 modificó la cantidad de Senadores por cada provincia y la Capital Federal de dos a tres senadores (CNA, 1994, art 54), pasando así de 48 escaños a 72 en total.

En Brasil el número de diputados federales es proporcional a la población ajustándose el porcentaje el año previo a la elección, no pudiendo haber más de 513 diputados y habiéndose alcanzado este número en la actualidad. Respecto de la Cámara de Senadores hay tres senadores por estado (CNB, 1988 art. 45 y 46 inc. 1), sumando 81 en total.

Se puede observar la cantidad de mujeres que componen las cámaras que se analizan, así como también cuántas de ellas son electas en cada proceso electoral en los Cuadros 2 y 3.

CUADRO 2

Diputadas en Argentina y Brasil, 1991-2019.

\begin{tabular}{|l|l|l|l|l|l|l|}
\hline \multicolumn{5}{|l|}{ DIPUTADAS } \\
\hline ARGENTINA & $1991-1993$ & $1993-1995$ & $2017-2019$ & $\begin{array}{l}1995- \\
1998\end{array}$ & $1999-2003$ & $2015-2019$ \\
\hline Periodo electoral & 254 & 257 & 257 & 513 & 513 & 513 \\
\hline $\begin{array}{l}\text { Cantidad total de } \\
\text { escaños }\end{array}$ & 16 & 41 & 98 & 42 & 39 & 62 \\
\hline $\begin{array}{l}\text { Cantidad de } \\
\text { mujeres en la } \\
\text { Cámara }\end{array}$ & 130 & 127 & 127 & 513 & 513 & 513 \\
\hline $\begin{array}{l}\text { Escaños } \\
\text { disputados }\end{array}$ & 6 & 27 & 47 & 34 & 28 & 56 \\
\hline Mujeres electas & 6 & & & & & \\
\hline
\end{tabular}

Cuadro de elaboración propia con base en los datos obtenidos de Cámara dos deputados, Quem São Os Deputados, Recuperado de http://www2.camara.leg.br/deputados/pesquisa, Congreso de la Nación Argentina, Información Parlamentaria del Departamento de Asuntos Parlamentarios, Informe especial 103 y de Congreso de la Nación Argentina, Diputados, recuperado de http://www.diputados.gov.ar/secparl/dmuseo/archivo-Parlamentario/diputados.html 
CUADRO 3

Senadoras en Argentina y Brasil, 1991-2019

\begin{tabular}{|c|c|c|c|c|c|c|}
\hline \multicolumn{7}{|c|}{ SENADO } \\
\hline \multicolumn{4}{|l|}{ ARGENTINA } & \multicolumn{3}{|c|}{ BRASIL } \\
\hline Periodo electoral & 1983-1992 & 1993-1994 & 2017-2019 & $\begin{array}{l}1995- \\
1998\end{array}$ & $1999-2003$ & 2015-2019 \\
\hline $\begin{array}{l}\text { Cantidad total de } \\
\text { escaños }\end{array}$ & 48 & 48 & 72 & 81 & 81 & 81 \\
\hline $\begin{array}{l}\text { Cantidad de } \\
\text { mujeres en la } \\
\text { Cámara }\end{array}$ & 4 & 2 & 30 & 8 & 9 & 13 \\
\hline $\begin{array}{l}\text { Escaños } \\
\text { disputados }\end{array}$ & $\begin{array}{l}\text { No hay } \\
\text { elecciones }\end{array}$ & $\begin{array}{l}\text { No hay } \\
\text { elecciones }\end{array}$ & 24 & 54 & 27 & 27 \\
\hline Mujeres electas & & & 11 & 5 & 1 & 5 \\
\hline
\end{tabular}

elaboración propia a partir de los datos obtenidos de: Congreso de la Nación, Dirección de Información Parlamentaria (1995). Informe mujer. Recuperado de http://www.hcdn.gob.ar/export/hcdn/secparl/dgral_ info_parlamentaria/dip/archivos/Mujer.pdf: Senado de la Nación Argentina, Senadores, recuperado de http ://www.senado.gov.ar/senadores/Historico/PeriodoResultado; y Senado de la Nación Argentina, Senadores, Legislaturas anteriores, recuperado de http://www25.senado.leg.br/web/senadores/legislaturas-anteriores

Vale la pena recalcar la diferencia de tamaño que existe entre la Cámara de Diputados brasileña y la argentina. Además, es interesante tener presente que en el caso de la mencionada cámara, la renovación en el caso de Brasil es del total, es decir que en cada elección se disputan los 513 escaños que la misma contiene mientras en Argentina la renovación es parcial, en elecciones celebradas cada dos años.

A causa de los contrastes mencionados, es importante evaluar en términos relativos, más que absolutos, las situaciones presentadas. Dentro de los periodos abordados, en Argentina, se ven resultados semejantes a los esperados tras la aplicación de cuotas de género. En 1993, las mujeres ocuparon más del doble de las bancas de la Cámara de Diputados que, en 1991, y para el 2017, se sextuplicó la presencia femenina. La aplicación de la cuota permitió que, en las elecciones de 1993, casi un cuarto de los escaños fuera ganados por mujeres, en el 2017, el doble de mujeres ganó escaños durante la jornada electoral.

El caso brasileño es bastante distinto. Al evaluar la cantidad de mujeres en la misma Cámara, se observa que se parte de 42 mujeres previamente a la aplicación de la cuota a 39; inmediatamente después, y tras 12 años, son 62 las mujeres ocupando escaños. La presencia femenina tras la aplicación de una cuota legal no logró ser mayor a un cuarto. Sin embargo, respecto del segundo periodo, es interesante destacar que en el 2015 pudieron acceder el doble de mujeres.

La disparidad mencionada respecto del tamaño en las Cámaras de Diputados no es tal en el caso de las Cámaras de Senadores. En ambos casos, durante el primer y segundo periodo seleccionado, el Senado se muestra casi totalmente masculino. En el caso de Brasil, esta característica se mantiene hasta el tercer periodo evaluado. En la Cámara de Senadores, Brasil tampoco obtiene una presencia femenina mayor a un cuarto de la cámara. Algo a destacar es que, en la elección del 2015, se renovó un tercio de la Cámara mostrando un aumento en la proporción de mujeres electas respecto al periodo anterior evaluado, siendo equiparable a la cantidad de mujeres electas en 1998 cuando se renovaron dos tercios del total. 
En Argentina se ve que en 2017 la cantidad de mujeres senadoras es más cercana a la paridad, como así también el número de mujeres que fueron electas en ese año, en relación al total de escaños disputados.

\section{EstruCTURA INSTITUCIONAL DE LAS CUOTAS DE GÉNERO}

Las cuotas de género pueden definirse como:

"Medidas correctivas encaminadas a subsanar las barreras que históricamente han enfrentado las mujeres para ejercer plenamente su derecho de postularse para el ejercicio de cargos electivos, y como tales poseen el propósito de estimular su inclusión sistemática, no sólo excepcional, en las instituciones representativas" (Marx, Borner y Caminotti, 2007, p.165).

Existen tipos diferentes de cuotas, entre ellas, cuotas voluntarias, escaños reservados y cuotas legales, siendo estas últimas las de aplicación en Latinoamérica (Tula, 2015), y por ello eje del presente trabajo. No obstante, no todas las leyes de cuotas son iguales, y aun cuando lo son, su impacto efectivo no es análogo en todos los países (Archenti y Tula, 2007, p. 188). La estructura institucional de las mismas en los casos de análisis da cuenta de ello. ${ }^{6}$

Tanto Argentina como Brasil fijaron en sus leyes de cuota (Ley N²4.012 de 1991 y Ley N9.504 de 1997, respectivamente) un piso del $30 \%$ de mujeres en las listas. Sin embargo, la legislación brasileña estableció un máximo del 70\% para los candidatos del mismo sexo (art. 10 inc. 3), mientras la normativa argentina no sólo no plantea ningún tipo de porcentaje tope (Decreto $\left.\mathrm{N}^{\circ} 1.246,2000\right)$ sino que además contempló un mandato de posición fuerte, debiendo las mujeres ocupar un lugar en las listas que les permita resultar electas (Decreto $\left.\mathrm{N}^{\circ} 451,2005\right)$, y sanciones en caso de incumplimiento de la ley (Decreto Reglamentario $\mathrm{N}^{\circ} 379$, 1993; Decreto $N^{\circ} 1.246,2000$, art 11).

Si bien Brasil no contempla penalizaciones por la transgresión de la ley de cuota, sí lo hace ante el incumplimiento de los porcentajes mínimos establecidos para la financiación de programas (5\%) orientados a la promoción de la participación femenina en los partidos políticos y del tiempo de la pauta electoral (10\%) para ser usado por mujeres/incentivar su participación (Ley $\mathrm{N}^{\circ} 12.034,2009$ ).

Si bien comprendemos a las estructuras de las cuotas como un aspecto clave de esta investigación, vemos que no sólo el voto popular es condicionante del acceso definitivo al poder (Archenti y Tula, 2007). No podemos dejar de comprender estas estructuras dentro de la cultura política que circunda el sistema electoral de cada Estado analizado y de la región en general.

\section{ConCLusión}

Si se mira el interior de ambos parlamentos y se considera la existencia de cuotas de género fundadas en ley con el objetivo primario de garantizar la participación de las mujeres en la contienda electoral, se halla un reflejo de la realidad que muestra que la participación que permite la cuota no implica garantías de acceso a los espacios de poder, ni mucho menos representación de género, en ninguna de sus dimensiones en sentido estricto. Esto se debe a que el objetivo de la aplicación de cuotas es meramente la participación de las mujeres en las listas y contiendas electorales.

A la hora de comparar la situación en Argentina y Brasil, si bien en ambos casos los porcentajes que se busca asegurar de participación de mujeres son similares, el contenido restante de sus respectivas normas, así como la estructura del sistema electoral y la propia configuración social, política y cultural de ambos países, impactan en su aplicación y efectividad de la ley, mostrando en la práctica resultados disímiles.

En el caso de Brasil, la falta de penalizaciones al incumplimiento de la cuota sumada al uso de listas cerradas no bloqueadas genera grandes distorsiones a los efectos esperados de la cuota de género, que en el caso de Argentina no se perciben. El incumplimiento de los porcentajes de presencia femenina en las listas por los partidos no es entendido como una traba para su acceso al poder; asimismo, el tipo de lista genera 
una competencia dual que en líneas generales es desventajosa para las mujeres que tienen que competir no sólo al interior de los partidos para aparecer en la lista, sino además, una vez en ella, con los varones que la integran por el voto popular, el consecuente acceso a los cargos y el financiamiento de las campañas. La incompatibilidad con el sistema electoral y de partidos dificulta la aplicación de la cuota legal y la obtención de los resultados esperados, siendo así necesario comenzar a plantear nuevos mecanismos de participación más allá de las cuotas de género.

La situación en Argentina es distinta, ya que el sistema electoral y las leyes complementarias favorecen la efectividad en la aplicación de cuotas legales que permiten ampliar la participación de las mujeres en los espacios de poder, generando nuevas lógicas y perspectivas tanto al interior de los mismos en particular, como en la sociedad en general. Se percibe que el porcentaje mínimo de participación planteado por la ley de cuota se transformó con el paso del tiempo en un techo de facto, sancionándose en noviembre de 2017 la Ley de Paridad (Ley N $\left.{ }^{\circ} 4.412\right)^{7}$ que busca subsanar la situación. Resulta destacable la forma en que esto tuvo lugar, a partir de una alianza multipartidaria de legisladoras que necesitaron persuadir a sus compañeros de bloque para lograr el apoyo.

A pesar de las diferencias entre los casos, la tensión entre participación y representación atraviesa la cuestión tanto en Argentina como en Brasil. Muestra de ello es el contraste entre la demografía electoral actual de ambos países, mayoritariamente femenina, y la constitución predominantemente masculina de los Congresos (sobre todo en el caso brasileño). Así se evidencia la inexistencia de una relación lineal en términos de género entre votantes y representantes, que se traduce en un electorado que no vota en función del género y tampoco demanda representación en estos términos, y una clase dirigente que, no obligada a rendir cuentas en este sentido, se desliga parcial o completamente de tal representación.

A lo largo del trabajo se procuró ahondar en la participación efectiva de las mujeres en la arena legislativa, y de este modo se dio con lo que Pitkin (1985) denominó representación descriptiva. Sin embargo, queda por preguntarse acerca de la segunda dimensión que aborda dicha autora. ¿ El aumento de la participación y acceso de las mujeres al Parlamento implica linealmente la representación sustantiva de este colectivo? A la luz de los acontecimientos recientes en estas democracias se puede formular, a priori, una respuesta: aumentar la presencia femenina en las cámaras, no implica automáticamente hacer de los Congresos, Congresos feministas.

Que la representación aparezca como el reverso de la participación no debe entenderse como una equiparación entre sus significados, ya que incurrir en ese error implicaría asumir que existe una homogeneidad entre las mujeres a partir de la existencia de una identidad femenina única, cuando en realidad estas son múltiples y construidas a partir de diversas variables que se conjugan y condicionan entre sí.

Evaluar el grado de aumento en la representación sustantiva a partir de la implementación de las cuotas como mecanismo institucional, cómo esta podría generar una mayor incidencia política de las mujeres, cuáles son las barreras socio-culturales que excluyen de la arena política, y cuáles son los mecanismos que permitirán fortalecer los liderazgos individuales y colectivos para alcanzar un mayor protagonismo, son tareas pendientes que requieren un futuro esfuerzo de investigación. Sin embargo, vale la pena resaltar, que comenzar a tender canales para la participación hizo vislumbrar un horizonte posible para el ejercicio pleno de los derechos civiles, políticos y económicos por parte de las mujeres, que constituye uno de los grandes desafíos de la modernidad en vistas a alcanzar una democracia más participativa, representativa, igualitaria e inclusiva.

\section{REFERENCIAS}

Archenti, N. y Tula, M. I. (2007). Cuotas de género y tipo de lista en América Latina. Opinião pública, 13(1), 185-218. Caminotti, M. (2013). La representación política de las mujeres en el período democrático. RevistaSAAP. 7 (2). Argentina: Universidad Nacional de San Martín, pp. 329-337. 
Caminotti, M. y Freidenberg, F. (2016). Federalismo electoral, fortaleza de las cuotas de género y representación política de las mujeres en los ámbitos subnacionales en Argentina y México. Revista mexicana de cienciaspolíticas $y$ sociales, 228, 121-144

Castañeda, M (coord). (2013). Perspectivas feministas para fortalecer los liderazgos de mujeres jóvenes. México D.F: Centro de Investigaciones Interdisciplinarias en Ciencias y Humanidades de la Universidad Nacional Autónoma de México.

Dahl, R. (1992). La democracia y sus críticos. Barcelona: Paidós.

Marx, J., Borner, J. y Caminotti, M. (2007). Las legisladoras: cupos de género y politica en Argentina y Brasil. Buenos Aires: Siglo XXI Editora Iberoamericana.

Nohlen, D. (1994). Sistemas electorales y partidos políticos. México: Fondo de Cultura Económica.

Pitkin, H. F. (1985). El concepto de representación. Madrid: Centro de Estudios Constitucionales.

Sartori, G. (1988). Teoría de la democracia. El debate contemporáneo. Buenos Aires: REI.

Sartori, G. (2014). ¿Quées la democracia? Madrid: Taurus.

Tula, M. I. (2015). Mujeres y política. Un panorama sobre la adopción de las cuotas de género y sus efectos en América Latina y Colombia. OPERA, 16, 9-33.

\section{Material Normativo}

Código Electoral Nacional. (1983) Recuperado de: http://servicios.infoleg.gob.ar/infolegInternet/anexos/15000-19 999/19442/texact.htm

Código Eleitoral[Ley N ${ }^{\circ}$ 4.737]. (1965) Recuperado de: http://www.tse.jus.br/legislacao/codigo-eleitoral/codigo-ele itoral-1/codigo-eleitoral-lei-nb0-4.737-de-15-de-julho-de-1965

Congreso de la Nación Argentina. (1991). Ley $N^{\circ}$ 24.012. Recuperado de: http://servicios.infoleg.gob.ar/infolegInt ernet/anexos/0-4999/411/norma.htm

Congreso de la Nación Argentina. (2009). Ley $N^{\circ}$ 26.571. Recuperado de: http://servicios.infoleg.gob.ar/infolegInte rnet/anexos/160000-164999/161453/texact.htm

Congreso Nacional del Brasil. (2009). Ley $N^{\circ}$ 12.034. Recuperado de: http://oig.cepal.org/sites/default/files/2009_ lei12034_bra.pdf

Constitución de la Nación Argentina. (1994). Recuperado de: http://servicios.infoleg.gob.ar/infolegInternet/anexo s/0-4999/804/norma.htm

Constitución de la República Federativa de Brasil. (1988). Recuperado de: http://pdba.georgetown.edu/Constitutio ns/Brazil/esp88.htm

Poder Ejecutivo Nacional. (1993). Decreto reglamentario $N^{\circ}$ 379. Recuperado de: http://www.saij.gob.ar/379-nacio nal-cupo-femenino-listas-candidato

Poder Ejecutivo Nacional. (2000). Decreto No 1.246. Recuperado de:http://servicios.infoleg.gob.ar/infolegInternet/ anexos/65000-69999/65634/norma.htm

Poder Ejecutivo Nacional. (2005). Decreto $N^{\circ} 451$. Recuperado de: http://servicios.infoleg.gob.ar/infolegInternet/a nexos/105000-109999/106102/norma.htm

\section{Notas}

1 Se hablará a lo largo de todo el trabajo del concepto de mujer basado en la división binaria del género, ya que es en esos términos que se plantean las leyes de cuota.

2 De Argentina, se tomarán las elecciones de 1991, 1993 y 2017. En el caso de Brasil, las de 1995, 1998 y 2015, alentando así un trabajo de comparación en doble dirección, no sólo entre los países, sino además al interior de cada uno en el tiempo.

3 Resulta importante señalar que la crisis político institucional que atraviesa Brasil desde 2016 constituye un golpe a la estabilidad democrática del país y de la región en general. En dicho año la presidenta Dilma Rousseff(PT) fue depuesta 
por medio de un fraudulento proceso de impeachment, durante el cual su condición de mujer fue piedra angular de los discursos opositores. Durante las últimas elecciones presidenciales, "Lula" Da Silva, principal candidato del PT y candidato con mayor intención de voto, fue encarcelado bajo cargos de una causa jurídicamente muy cuestionable. En dichas elecciones, accedió al poder Jair Bolsonaro mediante un discurso xenófobo, violento, misógino, homofóbico e intolerante, que pone en cuestión la vigencia de los derechos humanos en el país vecino.

4 Los datos para el año 2017 son estimativos obtenidos de sumar el número de electores y de votantes por provincia. Recuperado de: http://www.resultados.gob.ar/escrutinio/dat01/DDN01999A.htm

5 La información de Argentina es estimativa, en función de cruzar los datos de sexo y edad correspondiente al censo del decenio de cada elección. En el caso de Brasil la falta de correlación entre la cantidad de habilitadxs para votar y la división por género responde a que hay personas cuyo sexo no fue identificado por el Tribunal Superior Eleitoral.

6 Mariana Caminotti y Flavia Freidenberg (2016) plantean cinco puntos a evaluar de la estructura: tamaño de la cuota, mandato de posición (establecimiento del lugar que deben ocupar las mujeres en las listas), penalizaciones que plantea la ley ante su incumplimiento, alcance de la cuota (listas de suplentes, renovación de cargos) y válvulas de escape (previsión de lugares donde no se pueda aplicar).

7 La nueva ley en Argentina no logra escapar de la lógica binaria de género que anteriormente se mencionó. Esta es una de las grandes críticas que se puedan presentar tanto a la Ley de Paridad como a las cuotas de género presentes tanto en Argentina como en Brasil en los períodos evaluados. 